\title{
Identification and analysis of randomised controlled trials in nursing: a preliminary study
}

Centre for Health Economics, University of York

Nicky Cullum, research fellow

Correspondence to: Dr Nicky Cullum, Centre for Health Economics, University of York, York YO1 5DD.

Accepted for publication 23 October 1996

\begin{abstract}
Objectives-To describe preliminary work undertaken for development of a nursing contribution to the Cochrane Collaboration. To ascertain whether there are randomised controlled trials (RCTs) on nursing care which need to be identified for inclusion in systematic reviews of the effects of health care.
\end{abstract}

Design-Searches by Medline (1966-94) and by hand of 11 nursing research journals from inception to the end of 1994 to identify RCTs and systematic reviews; and a comparison of searches by hand and by Medline for three nursing research journals.

Main measures-Total number of RCTs identified and number of RCTs published in nursing journals; the sensitivity of Medline searches; and aspects of nursing care evaluated by RCT.

Results-The work is ongoing and 522 reports of RCTs and 20 systematic reviews of effectiveness have been identified so far. The sensitivity of Medline searches for RCTs in nursing journals is as low as $36 \%$ for one journal and the lack of reference to research design in the title or abstract was the main reason for the lack of sensitivity. Conclusions-There are RCTs that evaluate aspects of nursing care, and are published in nursing and non-nursing journals, and are largely undertaken by nurses. These must be reviewed in ongoing systematic reviews of the effects of health care (including those undertaken as part of the Cochrane Collaboration). Nursing journals must be hand searched to identify these studies as the lack of reference to study design in the titles and abstracts of nursing trials leads to poor indexing in electronic databases such as Medline.

(Quality in Health Care 1997;6:2-6)

Keywords: randomised controlled trials; nursing.

\section{Introduction}

Nurses make up the largest professional workforce in the United Kingdom National Health Service (NHS), deliver most of the "hands on" patient care, and account for more than one third of purchasing expenditure and nearly half the salary costs. ${ }^{1}$ Nursing must therefore be part of any drive to identify and deliver effective health care, but the extent to which nursing has knowledge of the effectiveness of its practices is unknown. Research in nursing has been undertaken since Florence Nightin- gale's pioneering studies, and in the 1950 s the first nursing research journal (Nursing Research) was launched in the United States. In the United Kingdom, the then Ministry of Health invited the Royal College of Nursing to undertake studies aimed at measuring the effectiveness of nursing care. ${ }^{2}$ A series of research projects was undertaken, funded jointly by the Department of Health and Social Security and the Royal College of Nursing, which sought to answer questions about the quality and effectiveness of nursing care in areas such as wound $\mathrm{care}^{3}$ and giving information. ${ }^{4}$

In the United Kingdom in 1972 the Briggs report ${ }^{5}$ recommended that nursing should become more research based and the volume of research activity in nursing has increased apace since then. What is not clear, however, is how much this research has contributed to a knowledge of effective nursing care; how much of it has been appropriately (and inappropriately) implemented; and what the size of the gap between research findings and actual practice is.

In 1989, the Department of Health's strategy for nursing ${ }^{6}$ stated that: "All clinical practice should be founded on up-to-date information and research findings; practitioners should be encouraged to identify the needs and opportunities for research presented by their work".

This clearly often fails, perhaps in part because the onus for keeping abreast with research and developments has always been placed firmly with the individual nurse, who is expected to ensure the delivery of research based care, despite the fact that it might not be possible to access research findings during the working day and who's critical appraisal skills may be less than adequate. ${ }^{7}$

The lack of a history of collation of research in nursing is probably also a major barrier to implementing research. In medicine the importance of using a scientific approach which minimises bias to derive reliable summaries of the research in a particular area has been recognised for some time. When the effects of a treatment are modest, a systematic review which combines the results of all the available studies may be essential to appreciate the beneficial effects of the treatment - the administration of corticosteroids to women in preterm labour is such an example. ${ }^{8}$ In medicine, Antman' provided empirical evidence of the dangers of relying on unsystematic reviews by showing the gulf between the recommendations of "experts" in textbooks and reviews, and the accumulated research. However, the number of systematic 
reviews on which nurses can draw for practice is not large and this was recognised by the Taskforce on the Strategy for Research in Nursing, Midwifery, and Health Visiting, ${ }^{1}$ who placed great emphasis on the need for a feasibility study of how best to undertake systematic overviews across the entire field of research in nursing. This recommendation was particularly timely as it was published at the same time that the United Kingdom Cochrane Centre was established in Oxford.

The United Kingdom Cochrane Centre was inaugurated in Oxford in 1992 and has since stimulated the development of the international Cochrane Collaboration, with participants all over the world. The Collaboration consists of individual experts representing a range of perspectives, disciplines, and healthcare professions with shared clinical interests collaborating to assemble and maintain systematic reviews of the effects of health care. ${ }^{10}$ Cochrane reviews are published in the Cochrane database of systematic reviews which itself is part of the Cochrane library; issue 2 (1996) of which contains 114 systematic reviews and 131 protocols of reviews under development. Given the major part played by nurses in delivery of health care, and their research history, the need for nursing involvement in such an initiative is self evident.

NURSING AND THE COCHRANE COLLABORATION Exploratory work to assess how best nursing might be represented in the Cochrane Collaboration began in November 1993 and forms the basis of this paper. A prerequisite of any systematic review is the identification of as great a proportion as possible of all relevant research. The randomised controlled trial (RCT) is widely acknowledged as providing the least biased estimate of the relative effects of alternative forms of care which do not have dramatic differential effects, and most Cochrane reviews confine themselves to reviewing the results of RCTs. A feasibility study was therefore undertaken to find if RCTs have often been used in the evaluation of nursing. Electronic searching of Medline and hand searching of nursing specialist journals were the focus of this initial work; nursing journals were chosen not because nursing research is only published in these journals, but because other searching activity within the Collaboration (such as that undertaken by Cochrane review groups on the topic specific journals in their domain and Cochrane Centres on the general health care literature) will capture many of those randomised controlled trials directly relevant to or conducted by nurses and published elsewhere.

The project aimed to establish whether there is a body of RCTs in nursing by: (a) developing a search strategy for the identification of randomised controlled trials (RCTs) which evaluate aspects of nursing; and (b) with a combination of electronic and hand searching, identifying as high a proportion as possible of these RCTs. It then aimed to make the RCTs identified available and accessible to those undertaking systematic reviews in health care through the Medline enhancement programme ${ }^{11}$ and by publication in the Cochrane controlled trials register.

A subsidiary objective of the project was to identify existing systematic reviews in the nursing literature.

\section{Materials and methods}

INCLUSION AND EXCLUSION CRITERIA

The inclusion and exclusion criteria of the Cochrane Collaboration were applied for the identification of RCTs (box 1). Reviews were regarded as potential systematic reviews if they made some attempt to describe the methods by which they were conducted.

Inclusion and exclusion criteria for what was regarded as nursing were not developed for this work as the strategy sought to be over rather than under inclusive, leaving final decisions of relevance to those who will use the identified RCTs in future systematic reviews.

\section{SEARCHES OF SCIENTIFIC LITERATURE}

Two main strategies are used by the Cochrane Collaboration to identify RCTs - namely, the searching of electronic databases and of journals by hand - and the same strategies were adopted for this project. Electronic databases such as Medline have the advantage of being very quick to search, but cannot be relied on to be comprehensive or accurately indexed. It has been known for some time that Medline searches alone are inadequate for retrieving even those RCTs published in journals which are indexed by Medline. ${ }^{11}$

However, despite its shortcomings, Medline covers most nursing research journals and, unlike many nursing bibliographic databases (including the Cumulative Index of Nursing and the Allied Health Literature), indexes

\section{Eligibility criteria}

RANDOMISED CONTROLLED TRIALS

Reports of trials were eligible for inclusion if, on the basis of the best available information, it was judged that the people (or other units) followed up in the trial were definitely or possibly assigned prospectively to one of two or more alternative forms of health care with:

- random allocation

or

- a quasirandom method of allocation (such as allocating alternate cases, date of birth, or case sheet number).

Reports of trials are included even when no results were presented or when results were limited to the analyses of baseline variables. SYSTEMATIC REVIEWS

The inclusion of a description of the methods by which a review was conducted was regarded as a crude indicator of a potential systematic review and such reviews were collected for further assessment by the National Health Service Centre for Reviews and Dissemination for possible inclusion in the Database of Reviews and Effectiveness.

Box 1 Eligibility criteria. 
studies by research design as well as research topic. Medline was therefore the focus for the development of an initial search strategy for the identification of RCTs and systematic reviews in nursing, and was used to develop a hierarchy for hand searching of the nursing journals with most RCTs.

Medical subject headings (MeSH terms) pertaining to anything which could be described as nursing were taken from the annotated MeSH Index for 1992 along with methodological MeSH terms likely to be used to index or describe an RCT or a systematic review. The $\mathrm{MeSH}$ terms were then used along with free text terms in the final Medline search strategy (box 2 depicts the Silver Platter version).

Although the electronic searching is itself not time consuming, the search strategy undertaken for the years 1988-94 inclusive generated a mean of 415 references a year, many of which did not have abstracts. Each citation was then examined, a copy of each paper that was deemed likely or possibly an RCT or systematic review was obtained and compared with the eligibility criteria.

Medline (Silver Platter) search strategy to identify RCTs in nursing (MeSH terms in small caps; free text terms in lower case).

1 NURSE-PATIENT-RELATIONS (allows no subheadings)

2 explode NURSING-CARE /all subheadings

3 PSYCHIATRIC-NURSING /all subheadings

4 explode NURSING /all subheadings

5 explode NURSING-RESEARCH /all subheadings

6 explode NURSES /all subheadings

7 SKILLED-NURSING-FACILITIES /all subheadings

8 COMMUNITY-HEALTH-NURSING /all subheadings

9 SCHOOL-NURSING /all subheadings

10 health visit $^{\star}$

11 midwi*

12 nurs ${ }^{\star}$

$13 \mathrm{SB}=$ NURSING

141 or 2 or 3 or 4 or 5 or 6 or 7 or 8 or 9 or 10 or 11 or 12 or 13

15 RANDOM-ALLOCATION (allows no subheadings)

16 RANDOMISED-CONTROLLED TRIALS/all subheadings

17 DOUBLE-BLIND METHOD (allows no subheadings)

18 META-ANALYSIS (allows no subheadings)

19 randomly or randomised or controlled

20 meta-analysis

21 systematic review

22 RANDOMISED-CONTROLLED-TRIAL in PT

23 CONTROLLED-TRIAL in PT

2415 or 16 or 17 or 18 or 19 or 20 or 21 or 22 or 23

2514 and 24

Box 2 Medline (silver platter) search strategy $(P T=$ publication type).
Systematic page by page hand searching was also undertaken of those nursing journals shown by the Medline search to publish many RCTs. Careful instructions were prepared for three volunteer hand searchers, who were instructed to search every page of a journal for reference to RCTs and systematic reviews in articles, abstracts, letters, short reports, or any other format. The limited availability of resources and hand searchers did not permit duplication of searching for the purpose of estimating agreement between searchers.

The sensitivity of the Medline search (the proportion of actual RCTs identified by the electronic search) was calculated for three of the hand searched journals that are indexed by Medline.

All trials identified and verified as eligible for inclusion were forwarded to the United Kingdom Cochrane Centre for inclusion in the Cochrane Controlled Trials Register; al potential systematic reviews were forwarded to the NHS Centre for Reviews and Dissemination to be considered for inclusion in the Database of Reviews of Effectiveness (also part of the Cochrane library).

\section{Results}

This work is ongoing and so far Medline has been searched between 1987 and 1994, and several journals with many RCTs have been completely hand searched from their inception to the end of 1994 (table 1).

To date, 522 reports of trials and 20 systematic reviews of effectiveness have been identified, however, both electronic and hand searching is far from complete. Of the $\mathbf{5 2 2}$ RCTs identified, 375 were published in specialist nursing journals. Randomised controlled trials evaluating aspects of nursing have been published since (at least) 1955.

Table 2 shows the sensitivity of Medline searches for three important nursing research journals. It ranged from $36 \%$ to $80 \%$.

Table 3 describes the main research areas considered in the first 426 RCTs identified. A more detailed analysis of the studies which consider their methodological quality and other variables is ongoing as a separate study.

\section{Discussion}

Nursing, along with the other healthcare professions must seek to establish the effectiveness of its practices. Nurses often make choices

Table 1 Fournals hand searched from inception to the end of 1994

\begin{tabular}{lc}
\hline & $\begin{array}{l}\text { RCTs } \\
\text { identified (n) }\end{array}$ \\
\hline Advances in Nursing Science & 5 \\
Applied Nursing Research & 6 \\
Clinical Nursing Research & 11 \\
Research in Nursing and Health & 55 \\
Nursing Research & 92 \\
fournal of Clinical Nursing & 1 \\
Nursing Science Quarterly & 3 \\
fournal of Advanced Nursing & 51 \\
International fournal of Nursing Studies & 28 \\
Nurse Anaesthesia & 11 \\
Seminars in Perioperative Nursing & 0 \\
\hline
\end{tabular}


Table 2 Sensitivity of Medline search for three nursing journals

\begin{tabular}{lll}
\hline & Total RCTs & $\begin{array}{l}\text { Sensitivity of } \\
\text { Medline search } \\
\text { (\%) }\end{array}$ \\
\hline fournal & 51 & 80 \\
\hline fournal of Advanced Nursing & 71 \\
$\begin{array}{l}\text { Research in Nursing and Health } \\
\text { International fournal of Nursing }\end{array}$ & 55 & 36 \\
\hline Studies & 28 & 36 \\
\hline
\end{tabular}

Table 3 The 10 most common themes considered in the first 426 RCTs identified in the nursing specialist literature

\begin{tabular}{ll}
\hline Research area & $\begin{array}{l}\text { Frequency of } \\
\text { RCTs }\end{array}$ \\
\hline Patient education & 75 \\
Midwifery or neonatal care & 65 \\
Nurse education & 55 \\
Cardiac rehabilitation or coronary and & \\
critical care & 34 \\
Postoperative care & 31 \\
Anxiety prevention or reduction & 27 \\
Pain & 26 \\
Preoperative care & 24 \\
Paediatric nursing & 23 \\
Health promotion & 18 \\
\hline
\end{tabular}

between different ways of managing patients' problems and these decisions should be informed by evidence from good quality comparative studies.

This work has shown for the first time that there are RCTs published in the general health and nursing publications which have the potential to contribute to our overall knowledge of the effects of health care.

Hand searching is essential to adequately identify these RCTs. Athough 500 of the trials identified by hand searching were indexed by Medline (the 22 which were not were published in the early days of journals which subsequently became covered by Medline), many were not picked up by the Medline search because of a lack of any reference to study design in the title or abstract of the paper. The challenge then is for the editors of journals which publish nursing research to ensure that the titles and abstracts of published research seek to inform about research design and not just research topic. The adoption by nurse researchers of the language of the social sciences may also contribute to studies being termed experiments or quasi-experiments and perhaps reducing the likelihood of them being indexed as RCTs in what is essentially a biomedical index. The need for hand searching clearly has resource implications which need to be considered by those undertaking systematic reviews-such as, within the Cochrane Collaboration - however, such hand searching needs only to be undertaken once. The fruits of this particular work are now available as part of the Cochrane Library, and the searching will be expanded and updated as funds become available.

It is clear, even at this early stage of searching, that RCTs as a design occur far less often in the pages of nursing research journals than in those of medicine. There are probably several explanations for this - including the relative youth of research as an activity in nursing and the cost of undertaking trials compared with the cost of surveys or small scale qualitative studies. Nevertheless, there is something of an "antitrial" culture in nursing in the United Kingdom, where nurses seem particularly keen to adopt research designs which are clearly different from their medical colleagues. Thus papers which argue for qualitative research in preference to the quantitative - for example, RCTs - abound. A recent article ${ }^{12}$ about action research in the Nursing Times (the most widely read nursing journal in the United Kingdom ) was printed under the banner "Double blind randomised trials are not the only way to gather data, nor even the best way" and others have argued that nursing is overdependent on science. ${ }^{13}$ This is despite the fact that a recent systematic review of research on pressure sore ${ }^{14}$ showed that a scientific basis for prevention of pressure sores (a core nursing activity) does not exist.

It is apparent from this work that nurses have a history of using experimental methods to evaluate the most fundamental of nursing activities, such as how we give information to patients, or help reduce anxiety in patients awaiting surgery. The shear breadth of topics considered with an RCT design is striking and the comparatively large proportion of trials evaluating methods of educating nurses is also noteworthy - possibly arising from the difficulties nurses have faced in obtaining approval to undertake research on patients.

The nursing literature contains many RCTs which should not be ignored; they are an essential component of the kind of multidisciplinary systematic reviews of the effects of health care being undertaken by the Cochrane Collaboration and others. If these reviews are to be systematic they must incorporate as large a proportion as possible of all the evidence, and this will require exhaustive searching of all healthcare literature, including nursing and therapy journals.

We need to know what research already tells us about the effectiveness of nursing interventions. This is necessary so that good practice can be promoted, and less effective or even harmful practices can be abandoned. Moreover we need a systematic approach to the identification of gaps in our knowledge so that the necessary research can be undertaken. The production of systematic reviews is an essential step towards promoting research based nursing practice, and the participation of nursing in the Cochrane Collaboration is important to ensure that the existing body of nursing knowledge is readily available and accessible within multiprofessional reviews focused on clinical topics. So far, relatively few nurses have volunteered themselves as Cochrane reviewers, despite the fact that involvement of nurses in review groups has been actively encouraged.

Finally, we need to convey the message that the drive to define effective nursing care does not aim to stifle the creative intuitive approach to care, but to foster a desire to properly evaluate new nursing interventions. As Oakley ${ }^{15}$ so eloquently puts it: "the condemnation of 
experimentation under the heading of "research" allows a great deal of experimentation to pass unnoticed under the heading of standard practice".

The proper evaluation of new (and established) developments in nursing practice can only enhance the status of nursing, and most importantly ensure that patients receive effective nursing care. Qualitative research will always be required to answer questions such as how illnesses impact on individual people, and could be used increasingly within RCTs to find out what patients think about new nursing developments or interventions. What is important is that nursing avoids falling into the trap of assuming that the rapid introduction of new nursing practices, without proper evaluation, is always in the best interests of the patient.

I acknowledge the Department of Health and North West Regional Health Authority for the initial funding of this work. Thanks are due to the volunteer hand searchers, Drs Joyce Prince and Kate Seers, and Ms Beverley French; to Mrs Lesley Ronald for the administration of searching and for the database; and to Drs Iain Chalmers and Anne Mulhall, and Professor Trevor Sheldon for their critiques of earlier drafts of this paper.

1 Department of Health. Report of the Taskforce on a Strategy for Research in Nursing, Midwifery and Health Visiting. London: Department of Health, 1993.
2 McFarlane JK. The proper study of the nurse. London: Royal College of Nursing 1970

3 Hunt JM. The teaching and practice of surgical dressings in three hospitals. London: Royal College of Nursing, 1974.

4 Hayward J. Information - a prescription against pain. London Royal College of Nursing, 1975.

5 Briggs A. Report of the Committee on Nursing. London: HMSO, 1972. (Cmnd 5115.)

6 Department of Health. A strategy for nursing: report of the Steering Committee. London: HMSO, 1989.

7 Pearcey PA. Achieving research-based nursing practice. $\mathscr{f}$ Adv Nurs 1995;22:33-9.

8 Crowley P, Chalmers I, Keirse MJNC. The effects of corticosteroid administration before preterm delivery: an overview of the evidence from controlled trials. Br $\mathcal{F}$ Obstet Gynaecol 1990;97:11-25.

9 Antman EM, Lau J, Kupelnick B, Mosteller F, Chalmers TC. A comparison of results of meta-analyses of randomized control trials and recommendations of clinical experts. $\mathcal{F} A M A ; 268: 240-8$.

10 Chalmers I. The Cochrane Collaboration - Preparing, maintaining, and disseminating systematic reviews of the

11 Dickersin K, Scherer R, Lefebvre C. Identifying relevan studies for systematic reviews. In: Chalmers I, Altman D, eds. Systematic reviews. London: BMJ Publishing Group 1995.

12 Gibbings S. Research methods. Informed action. Nursing Times 1993;89:28-31.

13 Hawthorne DL, Yurkovich NI. Science, technology, caring and the professions: are they compatible? $\mathcal{f} A d v$ Nurs and the professions:

14 Cullum N, Deeks JJ, Fletcher AW, Sheldon TA, Song F. Preventing and treating pressure sores. Quality in Health Care 1995;4:289-97.

15 Oakley A. Who's afraid of the randomized controlled trial? Some dilemmas of the scientific method and good research practice. Women and Health 1989;15:25-59. 